\title{
DOES GOOGLE FAVOUR ITS OWN PLATFORMS IN SEARCH VISIBILITY?
}

\author{
Nataša Krstić \\ Faculty of Media and \\ Communications (FMK), \\ Singidunum University, \\ Belgrade, Serbia
}

\begin{abstract}
:
Google is the most dominant search engine in the world. Due to its monopoly, it is often targeted by regulators, accused of imposing its services, platforms and bias algorithm. The paper aims to determine whether a presence on Google-owned platforms improves the website visibility on the search engine, expressed through the search engine optimisation (SEO) score. For this purpose, the web presence of eight private universities in Serbia on Google My Business, Google Maps, YouTube and Google Images were analysed with software screening and observation of the search engine result page. The research results confirmed that Google favours its platforms, as universities with a symbiotic presence on all of them recorded the highest SEO score. Furthermore, Google gives the highest priority to a verified Google My Business listing, as it signals the authority of the university web presence. Since Google uses more than 250 factors in website ranking, the findings should be interpreted with caution.
\end{abstract}

\section{Keywords:}

search engine, Google My Business, Google Maps, YouTube, Google Images.
Correspondence:

Nataša Krstić

e-mail:

natasa.krstic@fmk.edu.rs

\section{INTRODUCTION}

In the first decade of the new Millennium, which is considered as the early stage in the development of the internet, there was still a fierce competition between search engines (Goehler, Cader, \& Szu, 2006). In 2005, Google had almost half of the market share (46.2\%), Yahoo! over one-fifth (22.5\%), and next to them were a dozen other smaller players in the search engines arena - MSN, AOL, My Way, Netscape and others (Fan, 2005). As of 2014, the situation changed significantly. Yelp, which helps consumers find and review restaurants and other local services, stated in its European antitrust lawsuit that the results on Google's search engine favour Google Plus, its direct competitor (Kanter, 2014). Foundem, a vertical search engine for finding the lowest online prices also filed a lawsuit claiming that Google deliberately ranked its own products ahead of Foundem in search results and sought damages for its business losses (Southern, 2020). Furthermore, in 2017, the European Commission (EC) imposed a record fine of $€ 2.42$ billion on Google in the Google Search (Shopping) Case (Magali, 2018), deciding that Google abused its market dominance by giving prominent placement in search results to its own comparison-shopping service while demoting rival services (Kokkoris, 2018). Since 2017, the EC has fined Google with $€ 8.2$ billion for anti-competitive practices; abusive practices in online advertising, the monopolistic market dominance of its Android operating system and for blocking advertising rivals in AdSense (EC, 2019).

Recently, in May 2020, the shares of the parent company Alphabet dropped by two per cent after the report that there would be antitrust charges by the United States Justice Department in the forthcoming summer. 
The Department of Justice, which is also scrutinizing Google's ad technology, is focusing on how Google leverages its dominant search position to stifle competition.

Despite so many regulatory penalties for anti-competitive behaviour and intrusive privacy rules, "google it" has become a transitive verb (Merriam-Webster, n.d.) and Google (owned by Alphabet) is still over-dominant across the online ad ecosystem and as a search engine. In Serbia, Google holds $98.35 \%$ of the search market share, and globally $94 \%$ (Statcounter, n.d.). Only in China, South Korea, Russia and the Czech Republic, these numbers are lower. This clear monopoly comes not only from internet searches that go to Google (69.4\%) but also from those routed to its platforms: Google Images (20.5\%), YouTube (3\%), Google My Business and Google Maps (0.8\%) (Fishkin, 2019). Depending on the search query, Google News, Google Travel, Google Weather, Google Scholar might also steal some portion of web searches. Consequently, if Google's algorithm is biased toward its platforms, it is advisable for web administrators to position business presence on Google's own properties (Fishkin, 2018) thus helping Google to reach, harvest and understand a web presence (Askey \& Arlitsch, 2014).

Search engines present search results according to a broad range of factors. Google is said to employ more than 250 factors in its ranking algorithm, most of which are being held as closely guarded secrets (Cheng-Jye, Sheng-An \& Ting-Li, 2016). In order to understand the position of a website on the search engine, web administrators use in practice various search engine optimisation tools and software, aiming to simulate Google algorithms in order to understand the visibility, performance and authority of its website on the search engine. In that respect, search engine optimisation (SEO) is defined as the implementation of practices aimed at making the websites friendly to both humans and search engines, thus improving their visibility on the search engine results pages (Dickinson \& Smit, 2015).

\subsection{Search Engine Results Page (SERP)}

The starting point of any internet search is the need, expressed through the intention of the internet users. Modern search engines can respond to user queries with relevant results that are displayed in a particular order or rank on the search engine results page (SERP). Depending on the type of the query, the SERP can present organic and paid website results, featured snippets, related searches and vertical results (such as Google My Business listing with its Google Maps location, Google News, YouTube videos, Google Knowledge graph) (Juon, Greiling \& Buerkle, 2012).

Importantly enough, "the presentation of results on the SERPs heavily influences users' selection of certain results" (Höchstötter, \& Lewandowski, 2009, p. 1796).
The results that are within the visible area ("above the fold"), without the need for the user to scroll down the SERP, are the ones which gain most of the clicks. Consequently, most users do not look beyond the first page of the search results, which means that if a website does not appear there, it loses visibility, and with it a good portion of web traffic (Pérez-Montoro \& Codina, 2017).

In June 2019, a new paradigm occurred; over a half of browser-based searches on Google (50.3\%) resulted in zero-clicks to websites (Fishkin, 2019). Specifically, Google tries to respond to search queries, especially those that come from mobile devices, without clicking on any results. In other word, Google is trying to serve users with a direct answer to their query directly on the SERP. As a result, searches without any clicks and clicks routed to Google-owned properties are steadily increasing (ibid). There has been a shift in Google's behaviour, from a search engine that drives searchers to websites to find answers to queries, to a self-hosted answering and solutions machine (Fishkin, 2018).

\subsection{The importance of web presence for educational institutions}

In the second decade of the 21st century, web presence became crucial for educational institutions, as a means of scholarly communication, a distance learning tool, an open forum for community engagement, and universal showcase for attracting talents. As a result, visitors achieve greater success and satisfaction while performing tasks on university websites that have strong web presences (Peker, Kucukozer-Cavdar \& Cagiltay, 2016). In other words, the web presence for higher education institutions not only has a lead generation role, but it is also in the function of SERP branding. Brand SERPs are the set of results that a search engine shows to users when they search online using a brand's name as their keyword. Based on that, reviewing and improving the web presence of a higher education institution can serve as a catalyst to improve the transmission of messages about the mission, values, differentiators and strengths - making the educational experience in the institution trustworthy and unique.

For this reason, in addition to the traditional "Shanghai List", the "Webometric Ranking of World Universities" - the initiative of the Cybermetrics Lab research group of the Spanish National Research Council, has recently gained importance as well.

The Webometrics Ranking combines webometric (the volume of the web content: the number of web pages and files) with classic bibliometric indicators (visibility and impact of web publications per number of citations) for university web presence ranking, thus providing a mixed indicator of both academic and web presence excellency (Maasoumeh \& Mohammad, 2019). 


\section{METHODOLOGY}

The research methodology includes software screening and observation of Google search results on the SERP, on the sample consisting of eight private universities in Serbia: Singidunum, Megatrend, Privredna akademija, Metropolitan, Union, Union Nikola Tesla, Alfa and International University of Novi Pazar (NEAQA, 2020). The analysis included only private universities, as they are not beneficiaries of the state budget for education, which makes their online visibility and web presence one of the major contributing factors to the enrolment of a new generation of students.

Given the introductory considerations, from which it arises that Google, together with its owned platforms has absolute dominance in the search engine market, the following hypothesis was formulated: University websites that have a presence on Google platforms have a better SEO score.

The research aimed to detect whether Google favours its own platforms by providing websites that have optimised presence on them with better organic search visibility. The visibility of the websites in Google search is presented as a total SEO score, shown on a scale from 1 to 100 (with 1-40 signifying low performance, 41-69 with opportunities to improve, and over 70 being well optimised to both search engines and users) (Lucia, 2020). For its calculation, the SEO and website review tool Woorank has been deployed in May 2020. WooRank displays its SEO score given on any website evaluated, as a result of analysing key factors that impact SEO and visibility of a website.

When it comes to owned Google platforms, the following methodology was applied:

- Strategic presence on Google My Business (GMB): claimed or verified listing, confirmed by not showing the option "Claim this Business" or "Own this business" (Capper, 2019). It was presumed that universities, because of their authority and relevance in the education community, must verify their GMB listing as a legitimate business, thus preventing Google or even competitors (Hawkins, 2017) from publishing out-of-date or inaccurate information. Also, unverified GMB listings are usually positioned lower in the search results compared to verified ones, which is why they have less visibility (Search Engine Journal, 2018a).

- Google Maps: owning a pinned location.

- YouTube: channel owned by the university.

- Google Images: ratio of images that do not contain an alternative description (alt-text) in the total number of website images, analysed by means of the Screaming Frog crawler software. Images without descriptive text will not be visible in Google Images search results (Baker, 2018). Because Google recommends that most, if not all images on a website be described (Illyes, 2018), in the conducted research the Google Images platform was categorised as good (up to $25 \%$ of images missing alt-text), tolerant (25-35\% without alt-text) and poor performance (over $35 \%$ images with no alt-text).

For reciprocity, the data collected through software screening has been uploaded on the Mendeley data repository (DOI: 10.17632/s6wsftwkwd.1).

\section{RESULTS}

With the initial hypothesis in mind, the web presence of eight private universities on four relevant Google platforms was analysed. The universities that made up the sample were ranked first according to their SEO score, after which their presence on chosen Google platforms Google My Business, Google Maps, YouTube and Google Images was shown, the last column displaying the number of Google platforms on which there is a web presence.

Table 1. Web presence of university websites on Google platforms

\begin{tabular}{llcccccc}
\hline \multicolumn{1}{c}{ URL } & SEO score & $\begin{array}{c}\text { Verified } \\
\text { GMB }\end{array}$ & $\begin{array}{c}\text { Pinned } \\
\text { Google Map }\end{array}$ & $\begin{array}{c}\text { YouTube } \\
\text { channel }\end{array}$ & $\begin{array}{c}\text { Google } \\
\text { Images } \\
\text { (missing alt text) }\end{array}$ & $\begin{array}{c}\text { Number of } \\
\text { Google } \\
\text { platforms }\end{array}$ \\
\hline 1 & Metropolitan.ac.rs & 77 (good) & Yes & Yes & Yes & $23.86 \%$ (good) & 4 \\
\hline 2 & Singidunum.ac.rs & 73 (good) & Yes & Yes & Yes & $11.64 \%$ (good) & 4 \\
\hline 3 & Alfa.edu.rs & 69 (medium) & No & Yes & Yes & $0.61 \%$ (good) & 3 \\
\hline 4 & privrednaakademija.edu.rs & 64 (medium) & No & No & Yes & $35.12 \%$ (poor) & 1 \\
\hline 5 & Unionnikolatesla.edu.rs & 64 (medium) & No & Yes & No & $94.29 \%$ (poor) & 1 \\
\hline 6 & Megatrend.edu.rs & 59 (medium) & No & Yes & Yes & $57.78 \%$ (poor) & 2 \\
\hline 7 & Uninp.edu.rs & 55 (medium) & No & Yes & Yes & $36.04 \%$ (poor) & 2 \\
\hline 8 & Union.edu.rs & 48 (medium) & No & No & No & $42.11 \%$ (poor) & 0 \\
\hline
\end{tabular}

Source: Woorank (SEO score), Google SERP (Google My Business, Google Maps, YouTube), Google Images (Screaming Frog), $19-21$ May 2020. 
The conducted research indicates the following results:

- The benchmark universities per SEO score, Metropolitan and Singidunum, are present on all the analysed Google platforms. The verified Google My Business listing additionally boosts their SEO score as a signal of authority to Google (Allworth, 2020), which is particularly important for educational institutions. In this regard, it should be noted that Metropolitan has a fully verified GMB listing (without the option: "Own this business"), while Singidunum has a partially verified GMB listing (existence of the option: "Own this business", the possibility of external entry to the account, but access to it being denied by the web administrator the same day) (Google My Business Help, n.d.).

- Conversely, the university with the lowest SEO score, Union, does not have an optimized presence on any of the analysed Google platforms.

- A well-ranked follower according to the SEO score, Alfa, has a presence on three out of four Google platforms.

- Non-optimised images, which are consequently not present in Google Images search, contribute significantly to the SEO score of the analysed universities.

\section{CONCLUSIONS}

For decades, Google has been a force in the world of technology, society, and information quality, improving many aspects of people's lives around the globe. However, when it comes to building services outside of its core web search business, there is strong evidence that the company has leveraged its monopoly position in its attempt to compete in other sectors unfairly.

Based on the above findings, we can conclude that the synergy of several platforms provides better search visibility, expressed through the SEO score. And vice versa, universities without a presence on Google platforms turn to be low achievers in terms of the SEO score.

The interpretation of the results obtained in this way entails certain limitations. The most significant limitation is the fact that Google uses more than 250 factors in website ranking, and for a precise insight, the quality of optimisation of the analysed platforms should be considered. In particular, this refers to whether images are merely described or contribute to and strengthen the website keyword strategy, which makes them relevant to search queries. Furthermore, it is also relevant how many subscribers YouTube channels have, and how well the accounts themselves are optimized. For Google My Business listings, the number and quality of user reviews are important as well. And last but not the least, it should be taken into account how technically superior university websites are and what is their authority, expressed by the quality of backlinks and number of referrals.
However, since the topic of the paper was not the excellence of the SEO optimisation, but whether Google algorithm is favouring its platforms in search visibility, it can be concluded that the starting hypothesis was confirmed, having in mind that the best-ranked universities are present on all of the analysed Google platforms, which positively impacts their search visibility.

The identified limitations also provide recommendations for future research, which may include considering the authority of university websites by analysing their backlink structure and quality or the impact of their social networks in the period preceding the enrolment of a new generation of students.

\section{LITERATURE}

Allworth, S. (2020, June 15). How to Use Google My Business to Boost Your Content \& SEO. Search Engine Journal. Retrieved June 30, 2020 from https://www.searchenginejournal.com/google-my-business-content-seo/370717/

Askey, D. \& Arlitsch, K. (2014). Heeding the Signals: Applying Web Best Practices When Google Recommends. Journal of Library Administration, 55(1), 49-59. doi: 10.1080/01930826.2014.978685

Baker, L. (2018, October 29). How to Boost Your Visibility on Google Images. Search Engine Journal. Retrieved May 21, 2020 from https://www.searchenginejournal.com/howto-boost-your-visibility-on-google-images/275261/

Capper, T. (2019, September 25). Check Your Google My Business Verification Status. Online Ownership. Retrieved May 15, 2020 from https://onlineownership.com/checkyour-google-my-business-verification-status/

Cheng-Jye, L., Sheng-An, Y. \& Ting-Li, D.H. (2016). Estimating Google's search engine ranking function from a search engine optimization perspective. Online Information Review, (40)2, 239-255, doi: 10.1108/OIR-04-2015-0112.

Dickinson, Z. \& Smit, M. (2015). Being where the people are: the challenges and benefits of search engine visibility for public libraries. Library Hi Tech News, 32(10), 11-15. doi: 10.1108/LHTN-08-2015-0055.

European Commission. (2019, March 19). Antitrust: Commission fines Google $€ 1.49$ billion for abusive practices in online advertising. Press release no. IP/19/1770. Retrieved May 20, 2020 from https://ec.europa.eu/commission/ presscorner/detail/en/IP_19_1770

Fan, J. (2005, July 21). Double-digit growth in search seen by $A O L$ and ASK Jeeves from Q1 to Q2 2005, while top search players Google and YAHOO! maintain consistent growth. NIELSEN NetRatings Inc. Retrieved May 20, 2020 from http://www.nielsen-online.com/pr/pr_050721.pdf

Fishkin, R. (2019, August 13). Less than Half of Google Searches Now Result in a Click. Sparktoro Blog. Retrieved May 15, 2020 from https://sparktoro.com/blog/less-than-half-ofgoogle-searches-now-result-in-a-click/ 
Fishkin, R. (2018, October 2). On SERP SEO - The Infuriating Reality of Search's Future. Sparktoro Blog. Retrieved May 16, 2020 from https://sparktoro.com/blog/on-serp-seothe-infuriating-reality-of-searchs-future/

Goehler, S., Cader, M., \& Szu, H. (2006, April). Smart internet search engine through $6 \mathrm{~W}$. Proceedings of SPIE - The International Society for Optical Engineering, (1)6247. DOI: 10.1117/12.669053

Google My Business Help. (n.d.). Request ownership of a Business Profile. Retrieved June 30 from https://support. google.com/business/answer/4566671?hl=en

Hawkins, J. (2017, October 9). Yes, Competitors Can Edit Your Listing on Google My Business. Moz blog. Retrieved May 21, 2020 from https://moz.com/blog/competitors-editlisting-google-my-business

Höchstötter, N., \& Lewandowski, D. (2009). What Users See - Structures in Search Engine Results Pages. Information Sciences, 179(12), 1796-1812. https://doi.org/10.1016/j. ins.2009.01.028

Juon, C., Greiling, D., \& Buerkle, C. (2012). Internet Marketing Start to Finish. Indianapolis, Ind.

Kanter, J. (2014, July 8). Yelp Joins Critics of European Union Antitrust Settlement with Google. The New York Times. Retrieved May 20, 2020 from https://www.nytimes. com/2014/07/09/technology/yelp-joins-critics-of-european-union-settlement-with-google.html?_r=0

Kendall, B. \& McKinnon, J.D. (2020, May 15). Justice Department, State Attorneys General Likely to Bring Antitrust Lawsuits Against Google. The Wall Street Journal. Retrieved May 21, 2020 from https://www.wsj.com/ articles/justice-department-state-attorneys-generallikely-to-bring-antitrust-lawsuits-against-google$11589573622 ?$ mod=article_inline

Kokkoris, I. (2018). The Google Saga: Episode I. European Competition Journal, 14(2/3), 462-490. https://doi.org/ 10.1080/17441056.2018.1540457

Lucia. (2020, April 27). Everything About the WooRank Score. Woorank Help \& Support. Retrieved May 19, 2020 from https://help.woorank.com/hc/en-us/articles/360000685389Everything-About-the-WooRank-Score?mobile_site=true

Maasoumeh K. S. \& Mohammad J. (2019). An Analysis of the Impact of SEO on University Website Ranking. Iranian Journal of Information Processing \& Management, 34(4), 1799-1822. DOI: 10.1016/j.acalib.2020.102171.

Magali, E. (2018). Fining Google: A Missed Opportunity for Legal Certainty? European Competition Journal, 14(1), 129-151. https://doi.org/10.1080/17441056.2018.1460973

Merriam-Webster. (n.d.). google verb. In Merriam-Webster. com dictionary. Retrieved May 13, 2020, from https:// www.merriam-webster.com/dictionary/google

National Entity for Accreditation and Quality Assurance in Higher Education. (2020, May 5). Accreditation Outcomes. NEAQA. Retrieved May 5, 2020 from https:// www.nat.rs/en/accreditation-outcomes/
Peker, S., Kucukozer-Cavdar, S. \& Cagiltay, K. (2016). Exploring the relationship between web presence and web usability for universities: A case study from Turkey. Program: electronic library and information systems, 50(2), 157-174. https://doi.org/10.1108/PROG-04-2014-0024

Pérez-Montoro, M. \& Codina, L. (2017). Navigation Design and SEO for Content-Intensive Websites: A Guide for an Efficient Digital Communication. Chapter 5: 109-124. Chandos Publishing.

Search Engine Journal (2019). Essential SEO Tools for Agencies. SEJ books, Alpha Brand Media.

Search Engine Journal (2018a). Local SEO: The Definitive Guide to Improve Your Local Search Rankings. SEJ books. Retrieved May 19, 2020 from https://www.searchenginejournal.com/local-seo/

Search Engine Journal [Gary Illyes]. (2018, October 25). What Image Publishers Do to Find Success on Google Images [Video]. YouTube. https://youtu.be/natBo3GKAwc

SEO Spider. (n.d.). Screaming Frog (Version 13). Screaming Frog Ltd. Retrieved March 17, 2020 from https://www. screamingfrog.co.uk/seo-spider/

Southern, M. (2020, April 4). Google May Be Forced to Reveal its Search Algorithm to an SEO. Search Engine Journal. https:// www.searchenginejournal.com/google-may-be-forcedto-reveal-its-search-algorithm-to-an-seo/359536/

Statcounter. (n.d.). Search Engine Market Share in Serbia April 2020 [Data set]. GlobalStats. Retrieved May 28, 2020 from https://gs.statcounter.com/search-enginemarket-share/all/serbia

Website Review Tool \& SEO Checker. (n.d.). WooRank. Retrieved May 18, 2020 from https://www.woorank.com/ 